\title{
Diseño de un turbo código en condiciones de SNR altas utilizando tramas pequeñas
}

\section{A Turbo Code Design Using Small Size Frames for High SNR}

\author{
Lazcano-Salas S. \\ División de Ingeniería Eléctrica \\ Facultad de Ingeniería \\ Universidad Nacional Autónoma de México \\ E-mail:saul_lazcano@hotmail.com \\ García-Ugalde F.J. \\ División de Ingeniería Eléctrica \\ Facultad de Ingeniería \\ Universidad Nacional Autónoma de México \\ E-mail:fgarciau@servidor.unam.mx
}

(Recibido: enero de 2009; reevaluado: octubre de 2009; aceptado: febrero de 2010)

\section{Resumen}

En el contexto de la codificación de canal se han realizado diversos estudios buscando alcanzar el límite teórico de Shannon; en este sentido, los turbo códigos se acercan bastante al mencionado límite. En el presente trabajo se hace un estudio comparativo entre algunos entrelazadores recomendados para tamaños de tramas pequeñas $(N<1024)$ bajo condiciones de perforado de tasa compatible, así como una sugerencia para la metodología de perforado, buscando mejorar el desempeño del turbo codificador, en términos de la tasa de bits erróneos (BER), particularmente en la zona de piso deruido (errorfloor), correspondiente a relaciones señal a ruido (SNR) altas.

Descriptores: turbo códigos, tasa compatible, patrón de perforado, entrelazadores, piso de ruido.

\begin{abstract}
In the context of channel communications there have been several studies searching the theoretical Shannon's limit. In this sense, turbo codes performance is very close to this limit. In this work we make a comparative study of some good interleavers for short frame size $(N<1024)$ considering rate compatible puncturing. We recommend a special puncturing methodology trying to improve the bit error frame (BER) especially at the error floor region and high $S N R$
\end{abstract}

Keywords: Turbo codes, rate compatible, puncturing pattern, interleavers, error floor region.

\section{Introducción}

Dentro de un sistema digital de comunicaciones o de almacenamiento de datos, se debe considerar de manera formal el contar con una metodología que permita controlar el número de errores que pudiesen existir en la información recuperada (sea la información recibida o leída, según el caso). En este sentido, la 
DOI: http://dx.doi.org/10.22201/fi.25940732e.2010.11n4.040

Diseño de un turbo código en condiciones de SNR altas utilizando tramas pequeñas

codificación de canal para la corrección de errores proporciona los medios para dicho control de errores.

Las bases de la codificación para corrección de errores o codificación de canal, fueron definidas de manera formal en 1948, en el trabajo de Claude E. Shannon (1948). En su trabajo, Shannon demuestra de manera matemática que, para un canal gaussiano con ruido blanco aditivo (canal $A W G N$ ), con una relación señal a ruido $(S N R)$ dada y un ancho de banda $(W)$, la cantidad máxima de información $(R)$ libre de errores que puede transmitirse está limitada por la ecuación:

$$
R<W \log _{2}(1+S N R)
$$

A partir de esta ecuación se establece el objetivo de alcanzar por la codificación de canal, el diseño de un esquema de codificación de tasa $R$ que cumpla con la ecuación anterior, el cual permita alcanzar una probabilidad de error lo suficientemente baja. La búsqueda de este objetivo trajo consigo un gran desarrollo en el área de la codificación de canal en la constante búsqueda del límite teórico predicho por Shannon. En 1993, un nuevo esquema de codificación llamado turbo códigos (Berrou et al., 1993), aparece en el escenario de la codificación de canal, demostrando alcanzar un desempeño cercano al límite predicho por Shannon para un canal $A W G N$. Su gran desempeño ha creado un esquema de codificación ampliamente estudiado e implementado en aplicaciones que van desde comunicaciones por fibra óptica, módems ADSL, comunicaciones satelitales y espaciales, así como sistemas ópticos de almacenamiento (Vucetic et al., 2002).

Este trabajo integra secciones referentes a las dos grandes categorías de códigos correctores de errores, la parte constitutiva de un turbo código, la metodología de perforado para lograr un turbo código de tasa compatible, las técnicas de entrelazado utilizadas, los resultados de las simulaciones y sus conclusiones.

\section{Tipos de códigos}

La figura 1 muestra un sistema básico de comunicaciones digitales (Vucetic et al., 2002). El bloque marcado como codificación de canal se encarga esencialmente de añadir redundancia a la información, al convertir un bloque de mensaje de longitud $k$ en una palabra código de longitud $n(n>k)$. En el receptor, el decodificador utiliza la redundancia $(n-k)$ para detectar y corregir los posibles errores que la información recibida pudiese tener.

Los códigos correctores de errores pueden ser divididos en dos grandes grupos: códigos de bloque y códigos

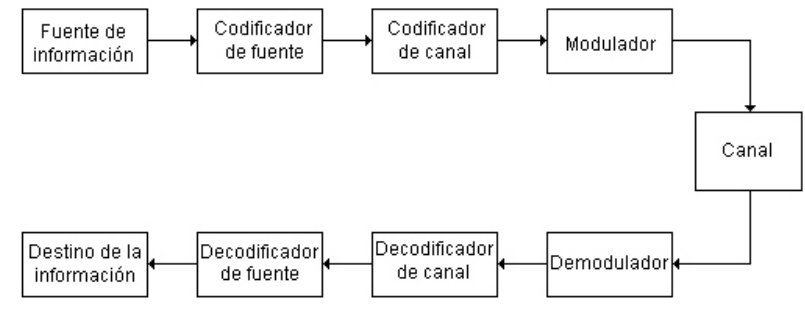

Figura 1. Esquema general de un sistema de comunicaciones digitales

de convolución. Entre los códigos de convolución y con el propósito de poder construir un turbo código con ellos, los más empleados son los códigos de convolución recursivos sistemáticos $(R S C)$. En un código sistemático, la secuencia de información de entrada se encuentra formando parte de la secuencia codificada de salida; los códigos de convolución sistemáticos presentan un mejor desempeño comparados con los no sistemáticos, siempre y cuando se realice una codificación recursiva (Vucetic et al., 2002).

El proceso de codificación puede ser realizado combinando dos o más tipos de códigos. Cuando la salida de un código llamado externo corresponde con la entrada de otro llamado interno, se le conoce como concatenación en serie, mientras que, si ambos códigos trabajan con la misma secuencia de información de entrada, cambiando simplemente el orden de los bits, se le conoce como concatenación en paralelo.

En varias aplicaciones que se encuentran en la literatura, la concatenación ha sido empleada en televisión de alta definición y en comunicaciones espaciales, por mencionar algunas (Morelos, 2006). Entre los esquemas de concatenación más populares se encuentra el que consiste en un codificador externo de bloque Reed - Solomon, conectado en serie con un codificador interno de convolución, separados por un entrelazador para disminuir la correlación entre los datos de entrada de cada codificador, como se observa en la figura 2.

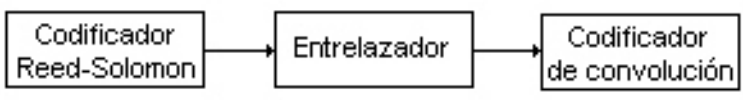

Figura 2. Concatenación en serie

Otro esquema más reciente de concatenación de códigos que ha tenido una aplicación muy amplia, debido a su capacidad de corrección de errores cercana al límite teórico de Shannon, es una concatenación en 
paralelo de dos o más códigos de convolución conectados entre sí, a través de un entrelazador (igualmente para disminuir la correlación de los datos de entrada), como se muestra en la figura 3. A esta configuración, definida en 1993, se le conoce como turbo códigos (Berrou et al., 1993).

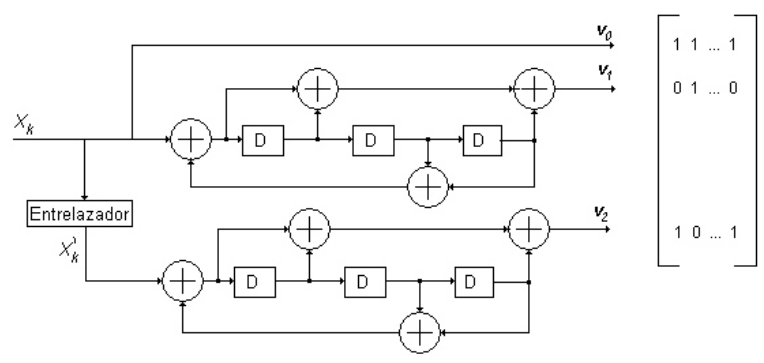

Figura 3. Esquema general de un turbo codificador con una matriz de perforado a la salida

\section{Elementos constitutivos de un turbo codificador}

De manera general, un turbo codificador se compone de un par de codificadores de convolución recursivos sistemáticos (RSC), un entrelazador y de manera opcional, una matriz de perforado para cambiar la tasa final del código, como se observa en la figura 3.

Los codificadores de convolución deben ser recursivos sistemáticos para que el decodificador pueda hacer uso de un intercambio iterativo de información. En estadística, este intercambio es conocido como "propagación de creencia" ("belief propagation"). En la figura 3, dentro de los codificadores, el símbolo "D" representa registros de corrimiento y las casillas marcadas con un símbolo "+" representan sumadores módulo 2. En esta figura, el codificador 1 (parte superior), trabaja directamente con la secuencia de entrada, simbolizada por $X_{k}$. Este primer codificador presenta dos salidas; la primera de ellas, $\boldsymbol{v}_{0}$, es igual a la secuencia de entrada, dado que los codificadores son sistemáticos. La otra salida, $\boldsymbol{v}_{\mathbf{1}}$, corresponde a la salida de paridad del codificador. Para reducir la correlación entre las entradas, los codificadores están conectados entre sí a través de un entrelazador, el cual puede ser definido brevemente como el proceso mediante el cual las secuencias de entrada y salida contienen los mismos elementos, pero en distinto orden; este cambio de ordenamiento puede ser realizado de manera determinista o aleatoria. Al proceso inverso se le llama desentrelazado y restaura la secuencia a su orden original (Vucetic et al., 2002). La primera función del entrelazador es construir un código de bloque de gran tamaño a partir de códigos de convolución de memoria pequeña. Al tener entonces un código de gran tamaño (ligado a $N$, longitud del entrelazador) se tiene entonces una mayor ganancia de codificación comparada con la ganancia obtenida con los códigos de convolución individuales. En teoría de códigos, es sabido que la probabilidad de bit erróneo, $P_{e}$, disminuye de manera proporcional al aumento del tamaño $N$. A este comportamiento se le conoce como ganancia de entrelazado . Otra función del entrelazador es cancelar la correlación entre las entradas de los dos codificadores RSC, para que en el decodificador sea posible aplicar un algoritmo iterativo de decodificación subóptimo, basado en un intercambio de información (Vucetic et al., 2002).

El codificador 2 (parte inferior) trabaja con una versión entrelazada de la secuencia original; esta versión entrelazada es denotada por $X_{k}^{\prime}$. Para este segundo codificador, únicamente la salida de paridad $\boldsymbol{v}_{2}$ es transmitida.

Algunas aplicaciones requieren códigos de tasas de codificación $\mathrm{R}=\mathrm{k} / \mathrm{n}$ más altas (es decir, códigos con menor redundancia), o incluso tasas adaptables al nivel de ruido del canal de comunicaciones, proceso conocido como RCPC ("Rate Compatible Punctured Codes"). Para lograr esto sin aumentar la complejidad del sistema se emplea una matriz de perforado, cuya función es determinar qué bits en determinadas posiciones serán, o no, transmitidos (posiciones marcadas en la matriz de perforado con "1" o "0", respectivamente, figura 3). Realizar perforado conlleva una adaptación en el desempeño del turbo código, ya que al enviar más o menos redundancia, la capacidad de corrección de errores aumenta o disminuye respectivamente, comparado con el turbo código original.

Esto se puede hacer de manera adaptable, dependiendo del nivel de ruido del canal y procurándose un conjunto de matrices de perforación, una de ellas será escogida por el transmisor como respuesta a una señal retroalimentada, enviada por el receptor (proceso adaptable) que mide el nivel de ruido del canal.

El perforado de tasa compatible (Hagenauer, 1988) conlleva la restricción de que los bits de salida del codificador de una tasa alta (poca redundancia, R cercana a la unidad) deban constituir un subconjunto de los bits de salida del codificador de una tasa menor (mayor redundancia, $R<1$ ). Dicho en otras palabras, los códigos de tasa alta están embebidos en los códigos de tasa menor, perteneciendo a la misma familia.

En la figura 3, la secuencia de información $\boldsymbol{v}_{0}$ y las salidas de paridad $\boldsymbol{v}_{1}$ y $\boldsymbol{v}_{2}$, son pasadas opcionalmente a través de una matriz de perforado en caso de necesitar reducir la cantidad de redundancia añadida y finalmente, son 
multiplexadas para generar la secuencia final del turbo codificador. Sin perforación, la tasa neta de codificación para el código de la figura es de $R=1 / 3$.

El alto desempeño, en términos de capacidad correctora de errores, se debe en gran medida al esquema de decodificación utilizado en la turbo decodificación. Un decodificador turbo se compone de dos decodificadores con los respectivos códigos RSC empleados en el codificador, separados por el mismo entrelazador (figura 4). Para poder sacar ventaja de la decodificación iterativa propia de los turbo decodificadores, la decodificación se basa en algoritmos de entrada y salida suave ("soft"), en donde la salida de un decodificador es la entrada del otro decodificador, el cual interpreta esta salida como información "a priori" para su proceso de decodificación.

Este intercambio de información entre ambos decodificadores se realiza de manera iterativa; a un mayor número de iteraciones en el proceso, la probabilidad de error de la decisión final del decodificador disminuye (proceso conocido en estadística como propagación de creencia "belief propagation"), permitiendo al turbo código alcanzar bajas probabilidades de bit erróneo $\left(P_{e}\right)$, del orden de $10^{-5}$, o $10^{-7}$ para relaciones señal a ruido (SNR) cercanas justamente al límite teórico de Shannon (1948).

Incrementar el número de iteraciones en el proceso iterativo tiene un comportamiento no lineal. Pasar de 2 a 6 iteraciones conlleva una ganancia de $1.7 \mathrm{~dB}$ mientras que, pasar de 6 a 18 iteraciones conlleva una ganancia menor de apenas $0.3 \mathrm{~dB}$ (Morelos, 2006), razón por la cual se recomienda un proceso de decodificación de entre 8 y 12 iteraciones.

En la figura 4 los vectores $\boldsymbol{r}_{0}, \boldsymbol{r}_{1}$ y $\boldsymbol{r}_{2}$ corresponden a los valores recibidos de los vectores transmitidos $\boldsymbol{v}_{0}, \boldsymbol{v}_{1} \mathrm{Y}$ $\boldsymbol{v}_{2}$ que fueron enviados por el transmisor. Los valores $\Lambda_{1 e}$ y $\Lambda_{2 e}$ corresponden a las salidas suaves de cada uno de los decodificadores.

Al cumplirse un criterio preestablecido, que puede ser un valor de $P_{e}$ objetivo, o un número máximo de iteraciones, el decodificador entrega una decisión binaria final sobre la información recibida, paso que se realiza por el comparador de signo de la salida en la figura 4.

\section{Metodología de perforado}

En este trabajo, para realizar el perforado se propuso una metodología que considera de manera explícita el tipo de entrelazador empleado, así como los codificadores de convolución RSC utilizados, de esta forma, se explotan mejor las características de cada elemento. Para valores de SNR de moderadas a altas, (superior a $2 \mathrm{~dB}$ ), casi todas las configuraciones de turbo codificadores presentan en sus curvas de desempeño $\left(B E R\right.$ vs $\left.P_{e}\right)$ un comportamiento de caída con menor pendiente, conocido como piso de ruido ("error floor"). En esta región, la distancia libre $\left(d_{\text {free }}\right)$ (Morelos, 2006) es el parámetro que determina principalmente el desempeño del turbo

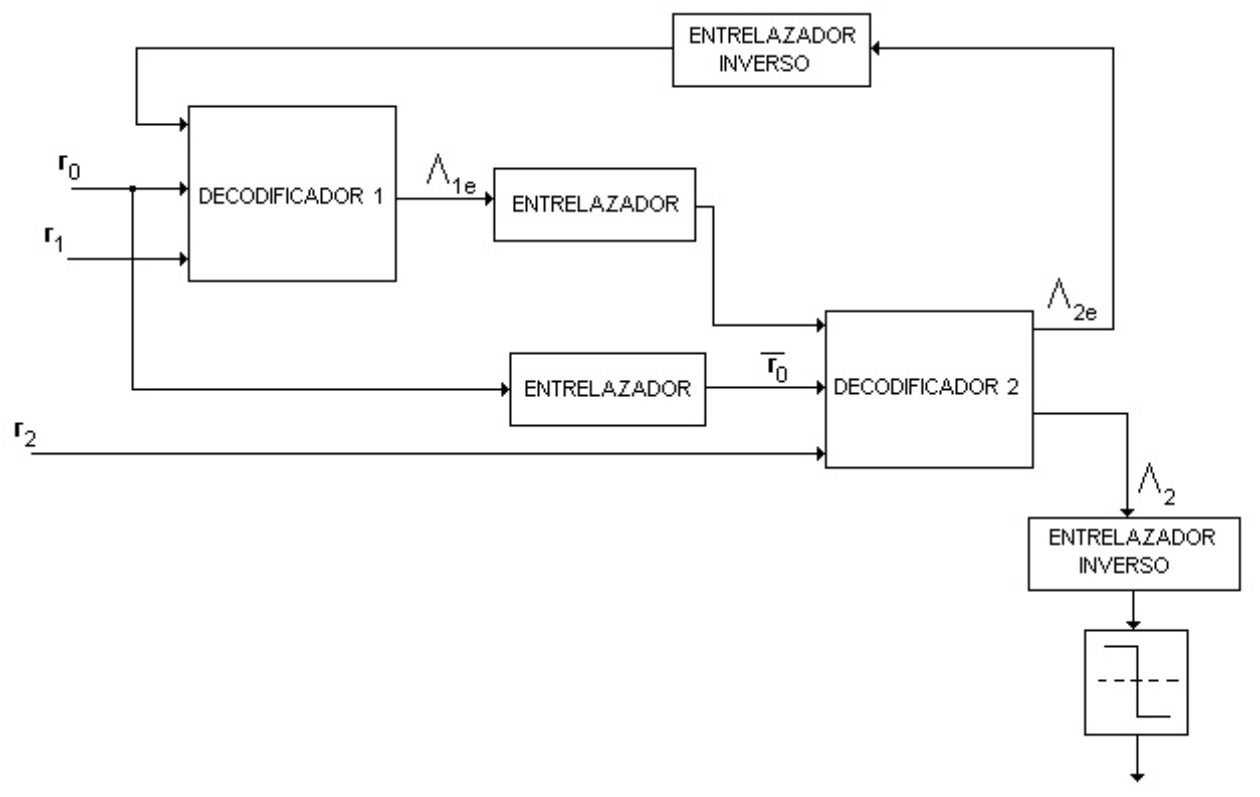

Figura 4 Esquema general de un turbo decodificador 
codificador. De acuerdo a lo anterior, en la metodología de perforado propuesta se busca que la $d_{\text {free }}$ se degrade lo menos posible al realizar el perforado.

El perforado se realiza de acuerdo a la siguiente metodología:

1. Se eligen los codificadores RSC. Para este estudio se eligen los codificadores RSC definidos por los polinomios en D $g_{0}(D)=1+D^{2}+D^{3} \quad \mathrm{y}$ $g_{1}(D)=1+D+D^{3}$ de acuerdo a (3GPPP, 2005).

2.Se elige un entrelazador. (La elección del entrelazador se describe en la siguiente sección).

3. Se elige el periodo de perforado y se construye la matriz de perforado, inicialmente con todas sus posiciones en valor " 1 ", es decir, sin perforar.

4. Se hace el análisis de las posiciones "candidatas" a ser perforadas de acuerdo al siguiente criterio: Sea $d_{w}$ el peso mínimo de palabras codificadas generadas por entradas de peso $w$, y sea $N_{w}$ el número de palabras código de peso $d_{w}$ (Babich et al., 2004). Para $w=2,\left(d_{w}, N_{w}\right)$ es igual a $\left(d_{\text {free }}\right.$, $N_{\text {free }}$, siendo $d_{\text {free }}$ la distancia libre del código convolucional, relacionada con la capacidad de corrección t del código, y $N_{\text {free }}$ el número de palabras código de peso $d_{\text {free }}$.

Se consideran como posiciones candidatas a ser perforadas únicamente las correspondientes a los bits de paridad de los codificadores 1 y 2 . Los bits sistemáticos del codificador 1 no se perforan (Babich et al., 2004).

De acuerdo a una restricción de tasa compatible (Hagenauer, 1988), se analiza cada posición candidata a ser perforada calculando sus duplas $\left(d_{w}, N_{w}\right)$. Se elige como posición a ser finalmente perforada, aquella que presente "la mejor" dupla $\left(d_{w}, N_{w}\right)$ es decir, $d_{w}$ debe ser el valor más alto (palabras de peso mínimo grande) y $N_{w}$ el menor valor presente (poca duplicidad de palabras del mismo peso). Para la obtención de las duplas $\left(d_{w}, N_{w}\right)$ se emplea el algoritmo propuesto en (Garello et al., 2001).

En caso de que exista más de un patrón de perforado con la misma dupla $\left(d_{w}, N_{w}\right)$, se procede a analizar el siguiente par $\left(d_{w+1}, N_{w+1}\right)$ para poder determinar el mejor patrón de perforado, lo anterior buscando mejorar el desempeño del turbo codificador en la región de interés, de valores SNR altos.

El paso 3 se realiza de manera recursiva, hasta alcanzar la tasa de codificación deseada. El diagrama de la figura 5 resume los pasos anteriormente descritos.

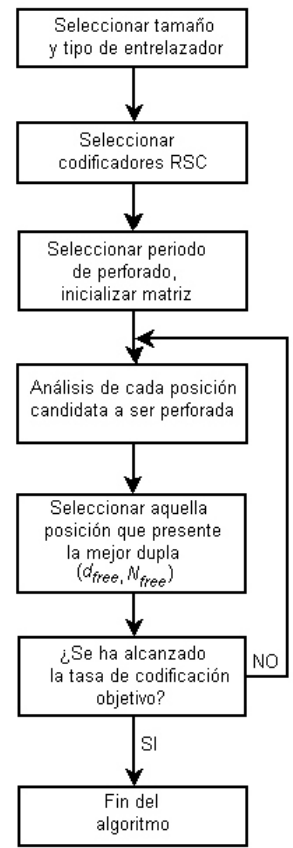

Figura 5 Diagrama de flujo, metodología de perforado

\section{Entrelazadores utilizados}

El entrelazador juega un papel muy importante dentro del desempeño del turbo código, su elección depende de diversos elementos y condiciones del sistema como la $S N R$, en la cual trabajará el sistema o el tamaño de trama $N$ elegido. Para relaciones señal a ruido $S N R$ bajas (canales con mucho ruido), cualquier entrelazador que garantice que las dos entradas a los codificadores tengan una correlación suficientemente baja, trabajará correctamente, mientras que para $S N R$ altas y debido a que en estas condiciones, el desempeño de un turbo código (TC) es dominado por las salidas de bajo peso del turbo código (afectan la $d_{\text {free }}$ ), por lo cual, una tarea adicional para el entrelazador es tratar de evitar dichas salidas de bajo peso, las cuales son generadas por respectivas entradas de bajo peso (Vucetic et al., 2002).

En este estudio se comparan los siguientes entrelazadores, considerando un tamaño de trama fijo de $N=256$ :

- El primer entrelazador es un entrelazador pseudoaleatorio, usado como referencia.

- El segundo entrelazador es un entrelazador denominado High Spread Random (HSR) (Crozier, 2000). La implementación del entrelazador HSR es similar a la del entrelazador S-random 
DOI: http://dx.doi.org/10.22201/fi.25940732e.2010.11n4.040

Diseño de un turbo código en condiciones de SNR altas utilizando tramas pequeñas

(Divsalar et al., 1995) con la variante de que se generan $N$ números reales aleatorios, en vez de $\mathrm{N}$ números enteros aleatorios. El entrelazador final se obtiene al ordenar los $N$ números reales aleatorios. El desempeño de este entrelazador en términos de la tasa de errores binarios (BER) es superior al del entrelazador $S$-random. Para este entrelazador, se elige una condición de esparcimiento de 20.

- El tercer entrelazador es el entrelazador llamado Dithered Relative Prime (DRP) (Crozier et al., 2001). Este entrelazador puede ser implementado en un proceso de 3 etapas en donde la salida de un proceso corresponde con la entrada del siguiente. En la primera etapa al vector de tamaño $N$ se le aplica una permutación de un periodo pequeño (comparado con $N$ ) utilizando un vector $\boldsymbol{r}$ de tamaño R, en donde $N=\mathrm{mR}$. En la segunda etapa, se realiza una permutación basada en números primos relativos para garantizar un esparcimiento adecuado. Finalmente, en la tercera etapa se realiza nuevamente una permutación de un periodo pequeño, ahora con un vector $\boldsymbol{w}$ de tamaño $\mathrm{W}$, en donde $\mathrm{N}=\mathrm{nW}$, con $n$ y m enteros.

- El cuarto entrelazador analizado es el entrelazador conocido como Golden (Crozier et al., 1999), cuya construcción involucra el ordenamiento de un vector de valores reales derivados de la "sección áurea". Para su construcción, se seleccionan los siguientes parámetros: $\mathrm{j}=0$ (podría ser cualquier entero módulo r), $\mathrm{m}=1$ (cualquier entero pequeño mayor a cero, los valores mas usados son 1 o 2) y r $=1$ (distancia entre elementos cercanos) (Crozier et al., 2001).

- El último entrelazador analizado es el denominado "Practical Size Interleaver" (PSI) (Wang et al., 2000), construido a partir de un entrelazador de bloque tradicional (las entradas son escritas en una matriz ordenadas por columnas y las salidas son leídas por renglones, o viceversa). Los datos de salida son reordenados en pequeños grupos, a los cuales se les aplican permutaciones inter-grupos y entre grupos, finalmente, los datos son leídos en orden inverso.

\section{Resultados}

Primeramente y como punto de referencia se comparan los entrelazadores utilizados, sin hacer una perforación en el código turbo. En la figura 6 se muestra el desempeño en términos de la tasa de errores binarios BER de los 5 entrelazadores comparados, para una tasa de codificación global de $R=\mathrm{k}_{0} / \mathrm{n}_{0}=1 / 3$. Como se observa en la figura mencionada, los 5 entrelazadores se comportan de manera parecida en la zona de caída de agua (waterfall); sin embargo, cuando se analiza la región de piso de ruido (error floor), aquellos entrelazadores que presentan la mejor dupla de valores $\left(d_{w}, N_{w}\right)$, mostrados en la tabla 1 (correspondiente a los entrelazadores DRP y PSI), son los que mejor desempeño presentan (menor atenuación en la pendiente de la curva, a medida que aumenta la razón señal a ruido $\mathrm{E}_{\mathrm{b}} / \mathrm{N}_{\mathrm{o}}$ ). Lo anterior, debido a su estructura determinística y a que su algoritmo de construcción, en ambos entrelazadores, considera de manera explícita los códigos RSC que componen el turbo codificador, obteniendo un entrelazador acoplado a los codificadores seleccionados.

En la teoría de códigos, es bien sabido que el proceso de perforado conlleva una degradación en el desempeño de la tasa de errores binarios (BER) de cualquier código. El caso de los turbo códigos no es la excepción y lo que se busca entonces es un patrón de perforado que degrade lo menos posible el desempeño del BER del código

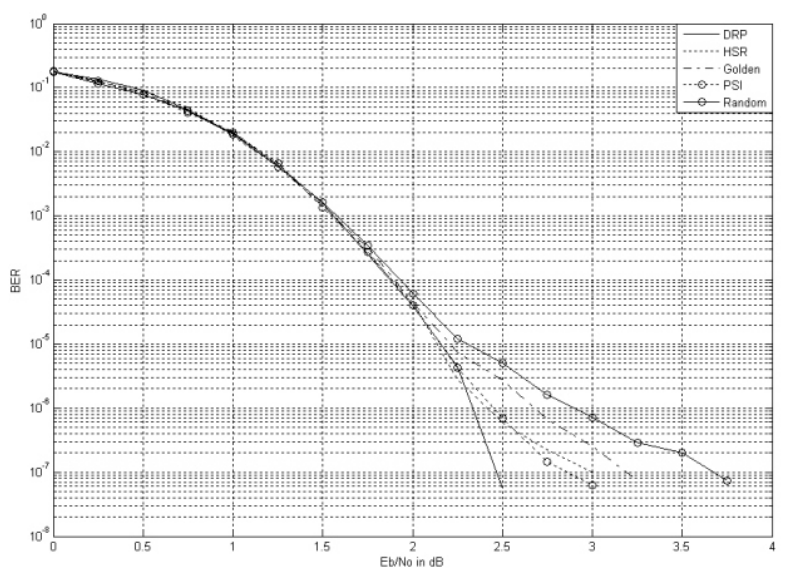

Figura 6. Desempeño de los entrelazadores seleccionados; para $R=1 / 3$

Tabla 1. Distancia libre y multiplicidad obtenidas para $R=1 / 3$

\begin{tabular}{cc}
\hline Entrelazador & $\left(d_{w}, N_{w}\right),\left(d_{w+1}, N_{w+1}\right),\left(d_{w+2}, N_{w+2}\right)$ \\
\hline DRP & $(20,2),(22,3),(23,4)$ \\
PSI & $(22,5),(23,4),(24,7)$ \\
HSR & $(18,14),(19,1),(20,1)$ \\
Golden & $(10,1),(20,2),(21,2)$ \\
Random & $(14,2),(16,3),(17,8)$ \\
\hline
\end{tabular}


resultante. Buscando cuidar el desempeño en la región de piso de ruido, se aplica la metodología de perforado anteriormente descrita, escogiendo "el mejor" patrón de perforado de tasa compatible, de acuerdo a la misma metodología. Dentro del perforado, otro elemento que afecta el desempeño es la estructura misma del entrelazador. A una mejor estructura, en el sentido de mejor esparcimiento, que cancele la correlación entre las secuencias de entrada y de salida y que disminuya las salidas de peso bajo, menor será la degradación que presente el turbo código resultante (Crozier et al., 2005).

Siguiendo la metodología de perforado presentada en este trabajo, se compararon los entrelazadores presentados en el párrafo anterior. La figura 7 muestra el desempeño de los entrelazadores analizados, ahora bajo condiciones de perforado que permitan una tasa global de codificación de $R=2 / 3$. Siguiendo la metodología, a cada entrelazador se le construye un patrón de perforado buscando la menor degradación posible, esto en comparación con el turbo código original. Así, cada entrelazador contó con su propio patrón de perforado. La tabla 2 muestra los pares de valores $\left(d_{w}, N_{w}\right)$ para la tasa de codificación buscada $R=2 / 3$. Nuevamente, los

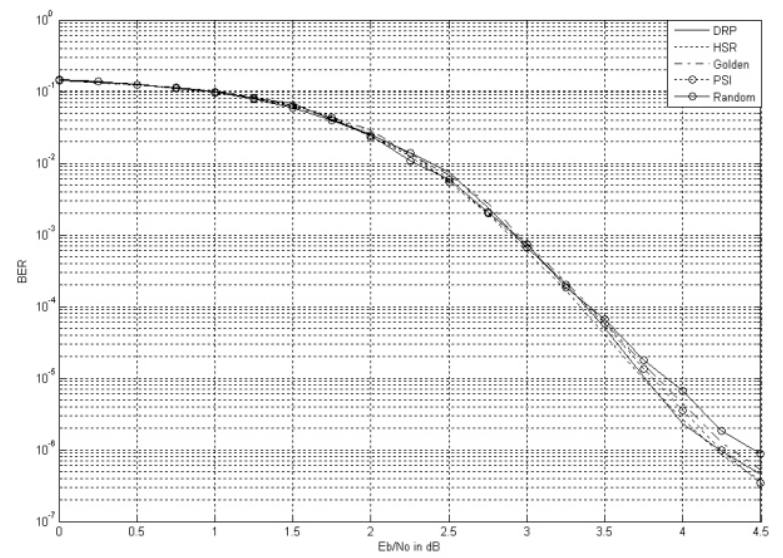

Figura 7. Desempeño de los entrelazadores seleccionados; para $R=2 / 3$

Tabla 2. Distancia libre y multiplicidad obtenidas para $R=2 / 3$

\begin{tabular}{ccc}
\hline Entrelazador & $\left(d_{w}, N_{w}\right),\left(d_{w+1}, N_{w+1}\right),\left(d_{w+2}, N_{w+2}\right)$ & Patrón perforado \\
\hline DRP & $(7,4),(8,30),(9,101)$ & $\left(1052_{\circ}, 20023_{\circ}\right)$ \\
PSI & $(7,19),(8,82),(9,272)$ & $\left(521_{\circ}, 61040_{\circ}\right)$ \\
HSR & $(6,9),(7,32),(8,62)$ & $\left(116644_{\circ}, 40575_{\circ}\right)$ \\
Golden & $(6,1),(7,11),(8,53)$ & $\left(24240_{\circ}, 11210_{\circ}\right)$ \\
Random & $(6,10),(7,26),(8,79)$ & $\left(22024_{\circ}, 60401_{\circ}\right)$ \\
\hline
\end{tabular}

entrelazadores con mejores duplas $\left(d_{w}, N_{w}\right)$ resultan en un desempeño ligeramente mejor que el resto de los entrelazadores. Los patrones de perforado son diseñados para cada entrelazador de manera explícita.

Teniendo cuidado con el cambio de escala, se pueden compara las figuras 6 y 7 , pudiendo observar que con la metodología propuesta el cambio de pendiente en las curvas para relaciones señal a ruido $\mathrm{E}_{\mathrm{b}} / \mathrm{N}_{\mathrm{o}}$ grandes, es menor (todos los entrelazadores utilizados tienen prácticamente el mismo desempeño en la figura 7, en la zona donde las curvas empezaban a separarse en la figura 6 , con lo cual se puede comprobar la aportación positiva de la metodología.

\section{Conclusiones}

A partir de la publicación en 1948 del trabajo de Claude E. Shannon, el esfuerzo realizado en la teoría de códigos en la búsqueda y desarrollo de un esquema de codificación que se acerque al el límite teórico de Shannon ha sido por demás extenso. En este sentido, los turbo códigos se acercan bastante a ese límite teórico, marcando así una nueva etapa en la historia de la codificación de canal.

En un turbo codificador, no existe una combinación de elementos que funcione, en general, mejor que el resto, sino que la elección de los elementos depende de las condiciones bajo las cuales debe operar el sistema, por ejemplo: tamaño de trama $N$, tipo y potencia del ruido del canal de comunicación, número de iteraciones, etc.

En Valenti (1999) se recomienda que la elección de los elementos se haga de manera conjunta buscando maximizar el buen desempeño global del turbo codificador. Bajo esta idea, en este trabajo se desarrolló una metodología de diseño y se hicieron pruebas a diferentes entrelazadores. Todos ellos tomando como referencia los codificadores de convolución elegidos (exceptuando el caso de los entrelazadores de naturaleza pseudoaleatoria.

En este trabajo, se propone una metodología de perforado, la cual busca que la degradación producida por el perforado sea la menor posible, principalmente en la región de piso de ruido y considerando además perforado de tasa compatible por razones prácticas, dado que en este caso cuando se cambia de una tasa a otra es más fácil la resincronización. La fortaleza de esta metodología radica en que se considera de una manera explícita, tanto los codificadores empleados, como el entrelazador utilizado. Obteniendo con esto, un patrón de perforado ligado al turbo codificador, buscando maximizar el desempeño del turbo codificador en su conjunto, particularmente en la región de piso de ruido, figuras 6 y 7 , 
zona en la cual un turbo codificador detiene el descenso en la curva de probabilidad de error. Por lo que cualquier mejora en el desempeño, sin aumentar la complejidad del sistema, es técnicamente deseable.

El realizar la construcción del turbo codificador, incluyendo al patrón de perforado, de manera acoplada, conlleva el poder explotar de una mejor manera las ventajas que cada elemento posee por separado, obteniendo un buen desempeño para las condiciones de operación globales consideradas. Con lo anterior, se pierde generalidad en el patrón de perforado obtenido; sin embargo, el patrón de perforado escogido, bajo la condición de tasa compatible, es el que menor degradación presenta para la combinación de elementos dentro del turbo codificador. Una mejora en el desempeño, concretamente en la región de relaciones SNR altas es por demás importante, considerando que la complejidad del sistema permanece sin cambios, ya que todo el trabajo se encamina a la construcción global del turbo codificador. Esta metodología puede aplicarse perfectamente en sistemas que requieran tasas de errores muy bajas y en sistemas de tasas de codificación adaptiva, gracias a la restricción de tasa compatible aplicada en la construcción del patrón de perforado.

\section{Agradecimientos}

Este trabajo se realizó gracias al apoyo del CONACyT México, proyecto 83694 y la DGAPA-UNAM, proyecto IN101305.

\section{Referencias}

3GPPP Technical Specification Group: Multiplexing and Channel Coding (TDD), TS 125.212V6.7.0. December 2005.

Babich F., Montorsi G., Vatta F. Some Notes on Rate-Compatible Punctured Turbo Codes (RCPTC) Design. IEEE Trans. Commun., 52(5):681-684, May 2004.

Berrou C., Glavieux A., Thitimajshida P. Near Shannon Limit Error-Correcting Coding and Decoding: Turbo codes. On:
Proc. IEEE Int. Conf. Communications, vol. 2, Geneva, Switzerland, 1993, pp. 1064-1070.

Crozier S. New High-Spread High-Distance Interleavers for Turbo-Codes. On: 20th Biennial Symposium on Communications, Kingston (Canada, May 28-31, 2000), Queen's University.

Crozier S., Guinand P. High Performance Low-Memory Interleavers Banks for Turbo Codes. On: IEEE 54th Vehicular Technology Conference, VTC Fall 2001 (October 7-11, 2001, Atlantic City, NJ, USA).

Crozier S., Lodge J., Guinand P. Hunt A. Performance of Turbo Codes with Relative Prime and Golden Interleaving Strategies. On: In 6th Intern. Mobile Satellite Conference (IMSC 99), (June 1999, Ottawa, Canada). Pp. 268-275.

Crozier S., Guinand P. Hunt A. On Designing Turbo Codes with Data Puncturing. On: 2005 Canadian Workshop on Information Theory (CWIT 2005) proceedings, (June 5-8 2005, Montreal, Quebec, Canada).

Divsalar D., Pollara F. Multiple Turbo Codes. On: MILCOM 95 (November 6-8, 1995). Pp. 279-285.

Garello R., Pierleoni P., Benedetto S. Computing the Free Distance of Turbo Codes and Serially Concatenated Codes with Interleavers: Algorithms and Applications. IEEE J. Select. Areas Commun., 19(5):800- 812, May 2001.

Hagenauer J. Rate-Compatible Punctured Convolutional Codes (RCPC Codes) and their Applications. IEEE Trans. on Communications, 36(4):389-400, April 1988.

Morelos-Zaragoza R.H. The Art of Error Correcting Coding. England. John Wiley \& Sons. 2006. 3GPPP Technical Specification Group: Multiplexing and Channel Coding (TDD), TS 125.212 V6.7.0. December 2005

Shannon C.E. A Mathematical Theory of Communication. Bell Sys. Tech. J., 27:379-423-623-656, 1948.

Vucetic B., Jinhong Y. Turbo Codes, Principles and Applications. Boston. Kluwer Academic Publishers. 2002.

Wang D., Kobayashi H. On Design of Interleavers with Practical Size for Turbo Codes. On: IEEE International Conference on Communications, (June 2000, ICC 2000). Vol. 2, 18-22.

Valenti M.C. Iterative Detection and Decoding for Wireless Communications (Ph.D. Dissertation), Bradley Dept. of Elect. \& Comp. Eng., Virginia Tech, July 1999. 
DOI: http://dx.doi.org/10.22201/fi.25940732e.2010.11n4.040

Lazcano-Salas S. y García-Ugalde F.J.

\section{Semblanza de los autores}

Saúl Lazcano-Salas. Obtuvo el título de ingeniero en telecomunicaciones por la Universidad Nacional Autónoma de México (UNAM) en 2000. Trabajó como ingeniero de desarrollo en Kb/TEL Telecomunicaciones de 1999 a 2001. Obtuvo el diplomado en gestión de las telecomunicaciones, Vodafone España y Escuela de Organización Industrial, 2002. Es profesor de asignatura en la División de Ciencias Básicas de la Facultad de Ingeniería, UNAM, desde Agosto 2007. Actualmente desarrolla el trabajo doctoral en la Facultad de Ingeniería, UNAM, sobre el tema de turbo codificación.

Francisco Javier García-Ugalde. Obtuvo su título como ingeniero mecánico electricista en el área de Ingeniería de Sistemas Eléctricos y Electrónicos de la UNAM en 1977. Su M.C. (Diplôme d'Ingénieur) en sistemas de comunicaciones/electrónicas del estado sólido por la Ecole Supérieure d'Electricité de Francia (SUPELEC) en 1980. Realizó su doctorado en la Universidad de Rennes I, Francia en 1982, en el área de Procesamiento de la Información. Desde 1983, es profesor de tiempo completo en la Facultad de Ingeniería de la UNAM. Sus intereses de investigación actuales son: Codificación y análisis de imágenes y video digitales, análisis, diseño y desarrollo de herramientas para filtros digitales, teoría y aplicaciones de codificación para el control de errores, codificación conjunta fuente-canal, turbo codificación, aplicaciones de criptografía, arquitectura de computadoras, procesamiento paralelo y bases de datos. 